\title{
Cardiac Arrest and Inverted Takotsubo Cardiomyopathy Following Intramyometrial Vasopressin Injection During Myomectomy
}

\author{
Alenka Golicnik ${ }^{1}$, Eda Vrtacnik Bokal ${ }^{2}$, Vojka Gorjup ${ }^{1}$ \\ ${ }^{1}$ Department for Intensive Internal Medicine, University Medical Center Ljubljana, Slovenia \\ ${ }^{2}$ Department for Reproduction, University Medical Center Ljubljana, Slovenia
}

Received: 22/03/2021

Accepted: $16 / 04 / 2021$

Published: $21 / 05 / 2021$

\begin{abstract}
How to cite this article: Golicnik A, Bokal EV, Gorjup V. Cardiac arrest and inverted takostubo cardiomyopathy following intramyometrial vasopressin injection during myomectomy. EJCRIM 2021;8: doi:10.12890/2021_002504.
\end{abstract}

Conflicts of Interests: The authors declare there are no competing interests.

This article is licensed under a Commons Attribution Non-Commercial 4.0 License

\section{ABSTRACT}

Vasopressin is involved in cardiovascular homeostasis that can influence coronary perfusion. It is commonly used as a local vasoconstricting agent during gynaecological procedures.

We present a case of cardiac arrest and inverse Takotsubo features following intramyometrial vasopressin administration during myomectomy. The patient was successfully resuscitated and recovered completely. Cardiac presentation was compatible with inverse Takotsubo cardiomyopathy that could have been triggered by high-dose vasopressin-induced coronary vasoconstriction. The patient's cardiac function resolved with no long-term sequelae.

Takotsubo cardiomyopathy usually results from an excessive catecholaminergic surge. High-dose vasopressin-induced coronary vasospasm could have been the mechanism underlying the clinical presentation in our patient.

\section{LEARNING POINTS}

- Local vasopressin administration during gynaecological procedures can result in rare but severe cardiovascular compromise.

- Takotsubo cardiomyopathy can result from multiple rare causes.

- High-dose vasopressin may cause Takotsubo cardiomyopathy features via coronary vasoconstriction.

\section{KEYWORDS}

Inverse Takotsubo cardiomyopathy, vasopressin, myomectomy

\section{INTRODUCTION}

Vasopressin is a hormone involved in maintenance of cardiovascular homeostasis that is also used in conditions ranging from diabetes insipidus to variceal bleeding and vasodilatory shock. Due to its vasoconstrictive effects, vasopressin is routinely used as a potent local vasoconstrictor in gynaecological procedures. However, local use of vasopressin during these procedures has been linked to rare but catastrophic complications such as severe bradycardia, cardiac arrest or pulmonary oedema ${ }^{[1]}$. These complications are associated with systemic absorption of vasopressin or inadvertent intravascular application. Vasopressin-induced increase in blood pressure is believed to cause a vagally mediated decrease in heart rate, while the vasoconstrictive effects of vasopressin may, simultaneously, cause coronary artery vasospasm ${ }^{[1]}$.

Takotsubo cardiomyopathy (TTC is a rare clinical entity occurring predominantly in postmenopausal women. It resembles acute coronary syndrome but with negative angiographic results. Echocardiography reveals transient cardiac apical akinesia/hypokinesia and basal 
hyperkinesia that resolves spontaneously ${ }^{[2]}$. It is believed to result from a catecholamine surge that leads, through multiple mechanisms, to myocardial damage ${ }^{[3]}$. Although only a small proportion (5.9\%) of TTC patients present with cardiac arrest, the majority of these patients had cardiac arrest as the first presentation to the medical service ${ }^{[4]}$. Different patterns of left ventricular (LV) dysfunction have been reported in TTC - the classic apical variant present in a high majority of cases, a midventricular variant and a basal or inverted variant ${ }^{[5]}$. Patients with inverted TTC tend to be younger; a triggering stress is usually present in patients with the classic variant, but almost always present in patients with the inverted variant ${ }^{[6]}$.

We present the case of a young woman who underwent a laparoscopic myomectomy that was complicated with a cardiac arrest following local vasopressin injection and who presented with an inverted TTC that resolved completely.

\section{CASE DESCRIPTION}

A 40-year-old female patient underwent a routine laparoscopic myomectomy for a single intramural myoma. Preoperative physical examination was unremarkable. After arrival to the operating theatre, the patient was monitored by 3-lead ECG, pulse oximetry and a non-invasive blood pressure monitor. Baseline vital parameters were normal. After premedication with propofol, fentanyl and rocuronium she was intubated. Total intravenous (IV) anaesthesia was continued with propofol and fentanyl and she was mechanically ventilated. Laparoscopic entry was uncomplicated and 8 units of vasopressin diluted to $0.1 \mathrm{U} / \mathrm{ml}$ were administered intramyometrially. An episode of sinus bradycardia with progression to asystole followed immediately after vasopressin administration. Cardiopulmonary resuscitation was started; the patient received $1 \mathrm{mg}$ of epinephrine IV and atropine $1 \mathrm{mg}$ IV. After 1 minute, spontaneous circulation was restored. The surgical procedure was terminated. Due to hypotension, the patient received boluses of phenylephrine 100 mcg IV and a continuous IV infusion of norepinephrine $0.25 \mathrm{mcg} / \mathrm{kg} / \mathrm{min}$. The patient was transferred to the ICU.

Afterwards the patient was haemodynamically and respiratory stable on low-dose norepinephrine and sedated with propofol. The ECG showed signs of ischaemia with ST segment depression in precordial leads; troponin levels were markedly elevated. Bedside transthoracic echocardiography was performed, which showed a normal-sized left ventricle with a moderately reduced ejection fraction and hypo/ akinesia of basal inferoposterior, lateral and interventricular segments. Coronary angiography was performed, which showed normal coronary arteries. Left ventriculography showed loss of basal segment contractility and apical hyperkinesia compatible with inverse TTC (iTTC) (Fig. 1).

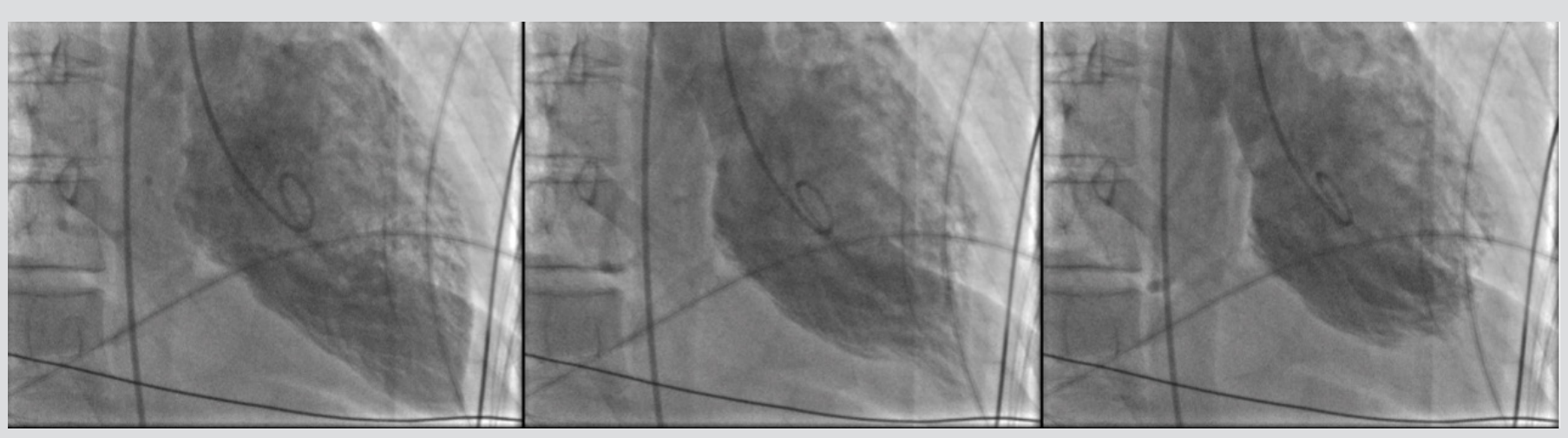

Figure 1. Invasive ventriculography demonstrating inverse Takotsubo features

The patient was stable and was weaned off the vasopressor. Control echocardiography performed after 6 hours showed less hypokinesia in the basal segments and improvement in the ejection fraction; ECG was normalized. On Day 2 sedation was discontinued, the patient awoke normally and was weaned off the ventilator. Troponin levels peaked on Day 2. She was transferred to a step-down unit at the cardiology department and after 3 days she was transferred back to the obstetrics department. She was put on medical therapy with aspirin, an ACE inhibitor and a beta blocker. Cardiac MRI was performed which showed a small area of transmural ischaemia inferolaterally. Echocardiography after 2 months showed complete resolution of hypokinesia and normal heart function.

\section{DISCUSSION}

It is speculated that adverse events following vasopressin application in gynaecological procedures are a consequence of an increase in systemic arterial pressure due to vasopressin-induced peripheral vasoconstriction, followed by reflex vagal bradycardia combined with 
coronary vasospasm and cardiac ischaemia ${ }^{[1]}$.

The vasopressin dose used in myomectomy is not uniform and varies in different reports. Due to the increased likelihood of adverse effects with higher dosages, a total dose of up to $5 U$ of dilute vasopressin is usually advised and can be repeated 45-60 minutes after the first administration ${ }^{[1]}$. Nevertheless, systemic absorption of such a dose is far higher than doses used for treatment of vasoplegic shock and therefore might result in coronary vasoconstriction and adverse effects.

In our case, the patient received $8 \mathrm{U}$ of vasopressin. As the injection was performed by an experienced operator inadvertent intravascular administration is unlikely, but the relatively high vasopressin dose used may have resulted in substantial systemic absorption.

Following a short resuscitation and haemodynamic stabilization, there were clear and persisting ECG signs of cardiac ischaemia and markedly increased troponin levels, which, after exclusion of coronary disease, we can not only attribute to transient cardiac ischaemia during the very short no-perfusion time, but we believe may have resulted from direct coronary vasoconstrictive effects of vasopressin as also seen in an animal model ${ }^{[7]}$.

The most interesting finding in our patient was the echocardiographic evidence of iTTC with a moderately reduced ejection fraction, which resolved completely during hospitalization. ITTC is a very rare entity, with an estimated incidence of $2.2 \%$ among cases of TTC and is usually seen in younger patients ${ }^{[8]}$. It is hypothesized that the presentation of inverted TTC at a young age could be due to the abundance of adrenoreceptors at the base compared to the apex in younger patients ${ }^{[6]}$. However, adrenoreceptor-mediated damage is only 1 of the several possible mechanisms implicated in TTC development. Epicardial and microvascular coronary vasoconstriction and/or spasm is another important mechanism believed to have a crucial role in TTC development ${ }^{[3]}$. High-dose vasopressin exerts vasoconstrictive effects on coronary vasculature ${ }^{[7]}$ and transient vasoconstriction and ischaemia in all 3 coronary regions could explain the observed iTTC features. To our knowledge, there are only limited reports on a possible association between vasopressin use and TTC occurrence ${ }^{[9]}$. Reports regarding adverse cardiac events following vasopressin use during gynaecological procedures usually do not include echocardiography. In our case, echocardiography was performed promptly after resuscitation and revealed iTTC features. Nevertheless, we cannot exclude the possibility that ITTC may have been the consequence of administration of epinephrine. However, global LV hypokinesia is a more frequent echocardiographic finding after cardiopulmonary resuscitation than TTC or ITTC, which leads us to speculate that high-dose vasopressin could have caused the iTTC features observed in our patient.

\section{REFERENCES}

Zeqja E, Qirko R. Vasopressine in laparoscopic myomectomy, a review over the effectiveness, dosage and possible complications. J Pharm 2016;6:23-27.

Dote K, Sato H, Tateishi H, Uchida T, Ishihara M. Myocardial stunning due to simultaneous multivessel coronary spasms: a review of 5 cases. J Cardiol 1991;21:203-214. Pelliccia F, Kaski JC, Crea F, Camici PG. Pathophysiology of Takotsubo syndrome. Circulation 2017;135(24):2426-2441.

Gili S, Cammann VL, Schlossbauer SA, Kato K, D'Ascenzo F, Di Vece D, et al. Cardiac arrest in takotsubo syndrome: results from the InterTAK Registry. Eur Heart J 2019;40:21422151.

5. Song BG, Chun WJ, Park YH, Kang GH, Oh J, Lee SC, et al. The clinical characteristics, laboratory parameters, electrocardiographic, and echocardiographic findings of reverse or inverted takotsubo cardiomyopathy: comparison with mid or apical variant. Clin Cardiol 2011;34:693-699.

6. Ramaraj R, Movahed MR. Reverse or inverted takotsubo cardiomyopathy (reverse left ventricular apical ballooning syndrome) presents at a younger age compared with the mid or apical variant and is always associated with triggering stress. Congest Heart Fail 2010;16:284-286.

7. Walker B, Childs M, Adams M. Direct cardiac effects of vasopressin: role of V1- and V2-vasopressinergic receptors. Am J Physiol 1988;255:H261-H265.

8. Ghadri JR, Cammann VL, Napp LC, Jurisic S, Diekmann J, Bataiosu DR, et al. Differences in the clinical profile and outcomes of typical and atypical takotsubo syndrome: data from the InternationalTakotsubo Registry. JAMA Cardiol 2016;1:335-340.

9. Littlejohn FC, Syed O, Ornstein E, Connolly ES, Heyer EJ. Takotsubo cardiomyopathy associated with anesthesia: three case reports. Cases J $2008 ; 1: 227$. 